\title{
Nanoscratch technique for aligning multiwalled carbon nanotubes synthesized by the arc discharge method in open air
}

\author{
A JOSEPH BERKMANS, M JAGANNATHAM and PRATHAP HARIDOSS* \\ Department of Metallurgical and Materials Engineering, Indian Institute of Technology Madras, Chennai 600036 , \\ Tamilnadu, India
}

MS received 26 December 2014; accepted 22 May 2015

\begin{abstract}
Horizontally aligned and densely packed multiwalled carbon nanotubes (MWCNTs) were synthesized in an open air, without the need for a controlled atmosphere, using a rotating cathode arc discharge method with the help of a metal scraper. The physical force exerted by the scraper results in in-situ alignment of MWCNTs along the direction of scrape marks. This strategy, which enables the alignment of nanotubes in a controlled fashion to any length and direction of interest, was examined to determine the force required to align a nanotube. A model is developed to understand the alignment process. Using the nanoscratch technique to mimic this strategy, and incorporating the data obtained from the nanoscratch technique into the model developed, the minimum force required to align a MWCNT, as well as the energy required to align a gram of nanotubes, has been estimated. The method demonstrated represents an economical approach for large-scale synthesis of aligned MWCNTs at low costs.
\end{abstract}

Keywords. Carbon nanotube; arc discharge; characterization; alignment; nanoscratch.

\section{Introduction}

Carbon nanotubes (CNTs) are technologically important materials due to their impressive physical and chemical properties and have been applied in various fields. Generally, CNTs can be synthesized by the arc discharge, ${ }^{1,2}$ chemical vapour deposition $(\mathrm{CVD})^{3}$ and laser ablation ${ }^{4}$ methods. CNTs are classified into single-walled carbon nanotubes $(\mathrm{SWCNTs})^{5}$ and multiwalled carbon nanotubes (MWCNTs) ${ }^{1}$ according to the number of concentric tubes present. Highly graphitized and relatively defect-free MWCNTs can be synthesized using the arc discharge technique, ${ }^{6}$ compared to CVD technique. Since the electrical and mechanical properties of the MWCNTs are superior along the tube axis, aligned MWCNTs are required in technological applications such as fabrication of electronic devices ${ }^{7,8}$ and composites. ${ }^{9-11}$ The aligned CNTs can be used to make high-performance and high-power flexible integrated circuits for electronic applications. Aligning CNTs in the polymer matrix enhances the thermal and electrical conductivity as well as strength of the composites. Horizontally aligned CNTs can also be used for interconnect applications. Obtaining aligned MWCNTs during, as well as after synthesis, at reduced costs has significant challenges. Vertical and horizontal alignment of CNTs is commonly attained by CVD techniques during synthesis ${ }^{12,13}$ and by post-treatments. ${ }^{14-16}$

Aligned MWCNTs using arc discharge under a controlled atmosphere of $\mathrm{N}_{2}$ has been demonstrated. ${ }^{17}$ The present work demonstrates in-situ synthesis of densely packed and

\footnotetext{
*Author for correspondence (prathap@iitm.ac.in)
}

horizontally aligned high-quality MWCNTs using the arc discharge method with the help of a metal scraper in an open air. This technique is extended after removing the soot from the cathode; physical forces are applied manually on the surface of the soot to align the MWCNTs along the direction of the application of the forces. Characterization techniques such as scanning electron microscopy (SEM) and transmission electron microscopy (TEM) have been carried out to observe the aligned CNTs. A model is developed to understand the forces and the energy required for the aligning process. The force required to align a single MWCNT, and the energy required to align a gram of nanotubes, is estimated by incorporating data obtained through the nanoscratch technique, into the model developed in this work.

\section{Experimental}

\subsection{Synthesizing MWCNTs}

A modified arc discharge set-up with a rotating cathode disc used to produce aligned MWCNTs in nitrogen atmosphere is described in detail elsewhere. ${ }^{17}$ In the present work, similar experimental conditions were used to synthesize aligned MWCNTs in a different atmosphere, open air, which has implications in terms of reduced cost and simplicity during synthesis. During arc discharge, when the gap between the electrodes is $\sim 1 \mathrm{~mm}$, the anode evaporates and deposits on the cathode as soot, and this soot contains MWCNTs.

The soot deposited on the cathode was continuously scraped by a metal scraper similar to a doctor blade with a 
length of $40 \mathrm{~mm}$, width of $20 \mathrm{~mm}$ and a thickness of $1 \mathrm{~mm}$, positioned close to the surface of the rotating cathode disc and away from the arcing region. Alignment was obtained by scraping the surface of the soot manually after it is removed from the cathode and is at room temperature, using a tip of a pair of tweezers with a tip diameter of $\sim 200 \mu \mathrm{m}$ and the flat side of a spatula.

\subsection{Nanoscratch test to align the synthesized MWCNTs}

Nanoscratch testing was performed across the aligned regions of the surface of the soot using a Triboindenter (Hysitron Inc., Minneapolis, USA). A Berkovich tip with a radius of curvature of 100-200 $\mathrm{nm}$ was used to scratch the surface by applying forces of 500, 750 and $1000 \mu \mathrm{N}$ up to a length of $20 \mu \mathrm{m}$, in independent experiments. The traverse speed of the tip was $0.67 \mu \mathrm{m} \mathrm{s}^{-1}$. The load and displacement resolution of the system are $3 \mu \mathrm{N}$ and $0.04 \mathrm{~nm}$, respectively. The same tip is used to scan the surface of the soot with a load of $2 \mu \mathrm{N}$ which results in scanning probe microscopy (SPM) micrographs.

\subsection{Characterization techniques used to analyse the alignment of MWCNTs}

FEI Inspect $\mathrm{F}$ field-emission scanning electron microscope (FE-SEM), with an accelerating voltage of $30 \mathrm{kV}$, was used to analyse the alignment of MWCNTs on the surface of the soot. FEI Quanta 200 scanning electron microscope (SEM) with a thermionic $\mathrm{LaB}_{6}$ filament, coupled with energydispersive spectroscopy (EDS) operated in secondary electron (SE) and back-scattered electron (BSE) modes, was used to locate the presence of nickel in the soot. Raman spectroscopic analysis (Model: WITec alpha 300 R) was carried out at room temperature with the laser wavelength of $532 \mathrm{~nm}$ on the aligned and unaligned regions of as-synthesized and purified soot samples. The as-synthesized soot scraped out from the cathode was characterized using PANalytical $\mathrm{X}$-ray diffractometer with $\mathrm{CuK}_{\alpha}$ radiation (the wavelength is $1.54 \AA$ ). Small quantities of MWCNTs taken out from the scraping marks were dispersed in ethanol using a CREST bath-type ultrasonicator of frequency $25 \mathrm{kHz}$ for $1 \mathrm{~min}$. A drop of the dispersed MWCNTs was placed on a carboncoated copper grid and analysed using transmission electron microscope (TEM). A Philips CM12 TEM with a LaB 6 filament at an acceleration voltage of $120 \mathrm{kV}$ was used to analyse the samples.

\section{Results and discussion}

\subsection{FE-SEM analysis of the aligned and unaligned surface of the CNTs}

The MWCNTs synthesized on the surface of the soot are shown in figure 1 . At lower magnifications, the scrape marks look smoother and flatter (white arrow) than the regular surfaces of the soot (black arrow), as shown in figure 1a. The regular surface contains highly entangled and randomly oriented MWCNTs present in the as-grown surface of the soot near the scraping marks shown in figure $1 \mathrm{~b}$. The magnified portion of the region of scraping mark is shown in figure 1c, where the scraping direction is indicated by a long black arrow. The surface is smoother and MWCNTs are closely packed, highly unentangled and aligned along the direction of the scraping mark (figure 1c and d) compared with the unaligned region as shown in figure $1 \mathrm{~b}$. On visual inspection of the micrograph, it is observed that finding a single and complete MWCNT with both its ends visible is highly unlikely in the unaligned region. However, in the aligned region most of the 'whole' MWCNTs can be clearly identified due to the un-entanglement during the application of the force. The force exerted by the scraper on the surface of the soot made scraping marks and the MWCNTs have got aligned due to this force. While MWCNTs are initially randomly oriented and entangled, when they become aligned they are all oriented in the same direction. Since MWCNTs are straight to begin with, and are now additionally pointed in the same direction, they become un-entangled. Following the in-situ aligning process on the soot, physical forces were manually applied on the surface of the soot after taking it out from the chamber at room temperature with the tip of a pair of tweezers; 'IIT' was inscribed on the soot. The different portions of the letters in the word were observed under FE-SEM, which clearly indicates the alignment of MWCNTs (figure 2) similar to the alignment obtained in the in-situ scraping marks.

The MWCNTs in the unaligned region is highly agglomerated and entangled as shown in figure 2a. FE-SEM analysis of the middle and bottom portions of the first alphabet 'I' of the word 'IIT' showing aligned MWCNTs, and they oriented along the direction of the inscription as shown in figure $2 b-d$, respectively. The MWCNTs in this region are significantly oriented and are relatively unentangled compared with unaligned region shown in figure $2 \mathrm{a}$. The region near the middle portion of the ' $\mathrm{I}$ ' is labelled as ' $\mathrm{a}$ ' and different regions in the word 'IIT' was labelled as $b-c$ and $f-i$, shown in figure $2 \mathrm{e}$. The magnified view of figure $2 \mathrm{c}$ is shown in figure $2 \mathrm{~d}$. The middle and bottom portions of the second letter 'I' contain aligned MWCNTs shown in figure $2 \mathrm{f}$ and $\mathrm{g}$, respectively. As similar to the previous micrographs, the MWCNTs are aligned along the direction of the scratch on top and middle portions of the third letter ' $\mathrm{T}$ ' as shown in figure $2 \mathrm{~h}$ and i, respectively. From FE-SEM micrographs, it is evident that the MWCNTs are aligned along the direction of the force applied and that they can be manipulated to align in different directions.

\subsection{Dimensions and misorientation of MWCNTs}

The length and diameter distribution of MWCNTs are calculated by measuring the individual length and diameter of 

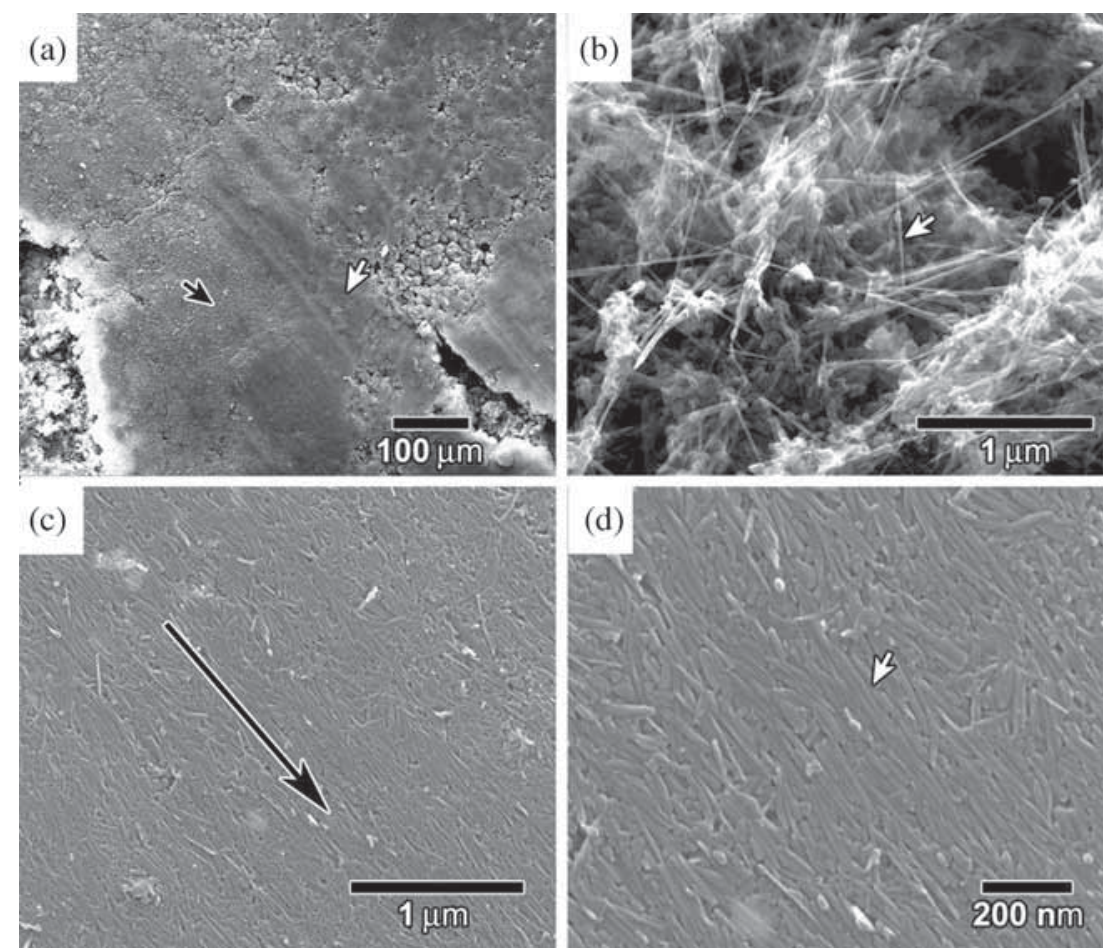

Figure 1. FE-SEM micrographs of in-situ scraped (white arrow) and unscraped (black arrow) regions on the surface of the soot (a). Unaligned MWCNTs present in the unscraped region indicated by a white arrow (b). Scrape mark at lower magnification, containing aligned MWCNTs and the alignment direction is indicated by a long black arrow (c). Scrape marks at higher magnification showing individual MWCNTs aligned along the direction of scraping marks, indicated by a white arrow (d).

MWCNTs from several FE-SEM micrographs and fitting the data obtained with a Gaussian distribution function. Using full-width and half-maximum (FWHM), the average length and diameter distributions of MWCNTs are calculated as $\sim 329$ and $\sim 23 \mathrm{~nm}$, respectively, as shown in figure $3 \mathrm{a}$ and $\mathrm{b}$. The mean value of the length and diameter of MWCNTs are $\sim 481$ and $\sim 24 \mathrm{~nm}$. The misorientation angle of the MWCNTs from the direction of aligning is calculated by considering an aligned MWCNT as a reference along the aligning direction. The order parameter of the aligned nanotubes is calculated from the equation $S=\left\langle 2 \cos ^{2} \theta-1\right\rangle,{ }^{18}$ where $\theta$ is the average misorientation angle. The calculated order parameter from figure $3 \mathrm{c}$ is $\sim 0.96$, which clearly indicates that significant alignment of MWCNTs has been attained by the application of physical forces. From the histogram, it is observed, $\sim 26 \%$ of MWCNTs are misoriented by $<10^{\circ}$ and $\sim 15 \%$ of them are misoriented by higher angles $>10^{\circ}$. The rest of the nanotubes are in the direction of alignment, without deviation as shown in the histogram (figure 3c). The average number of MWCNTs per micrometre length is $\sim 28$, and represents a densely packed alignment as shown in figure $3 \mathrm{~d}$. It is observed that most of the shorter nanotubes display greater deviation from the aligning direction than the longer ones, indicated by black arrows in figure 4 , similar to the thinner and shorter unaligned MWCNTs observed while cutting a polymer-resin nanotube composite. ${ }^{9}$ The deviation of the shorter nanotubes ranges from 17 to $75.1^{\circ}$. This poor alignment of shorter nanotubes can be attributed to the decreased duration over which the applied force acts on these smaller nanotubes relative to the larger one during aligning.

However, it is also seen that some of the shorter nanotubes are significantly aligned due to the forces exerted by the other nearby nanotubes during alignment, indicated by white arrows in figure 4. In general, from the experiments, it is seen that, the nanotubes should have sufficient length to be significantly oriented along the direction of applied forces and this may be easier in the case of SWCNTs due to their higher aspect ratio.

The packing density of the MWCNTs in the aligned region depends on the diameter of the nanotubes $\left(D_{\mathrm{M}}\right)$ and number of nanotubes $(N)$ in length $\left(L_{\mathrm{A}}\right)$. The packing density $(P)$ of the MWCNTs can be calculated as

$$
P=\left(D_{\mathrm{M}} \times N\right) / L_{\mathrm{A}} \times 100,
$$

$L_{\mathrm{A}}$ is considered as $1 \mu \mathrm{m}$. The packing efficiency of the aligned MWCNTs shown in figure $3 \mathrm{~d}$ is $\sim 64 \%$.

Manipulation of MWCNTs can be made by using the simple scratching method employed on the surface of the soot as shown in figure 5 . The as-synthesized soot contains no scraping marks and the surface of the soot is gently scraped manually after taking it out from the chamber, using a 

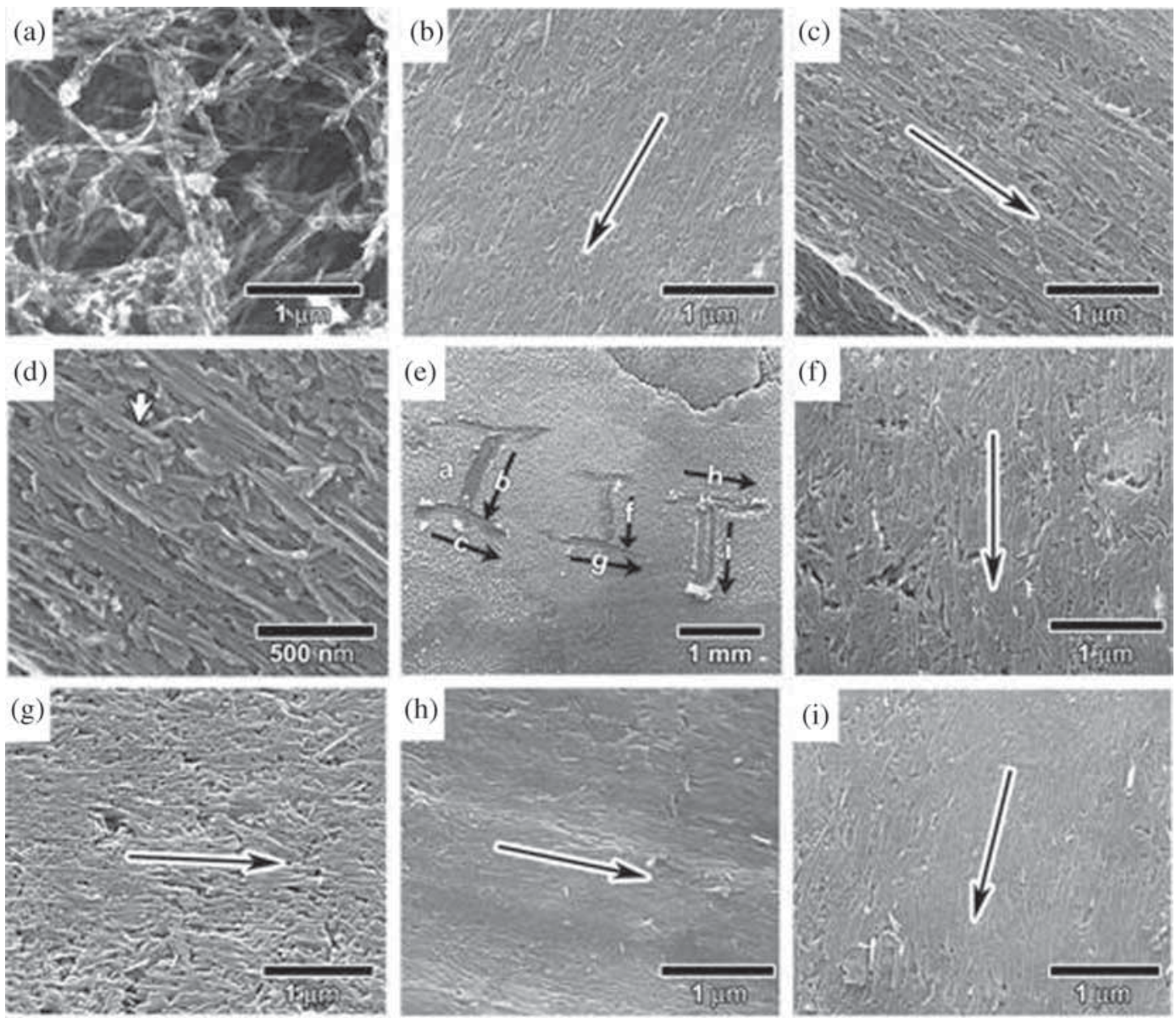

Figure 2. FE-SEM micrographs of unaligned MWCNTs near the letter 'I' (a). Middle and bottom portions of 'I' with aligned MWCNTs (b and $\mathbf{c}$ ). Higher magnification of figure $3 \mathrm{c}$ shows the MWCNTs clearly, indicated by a white arrow (d). Word 'IIT' inscribed on the soot with the tip of a pair of tweezers and the direction of inscription is indicated by arrows (e). Middle (f) and bottom (g) portions of second 'I' with aligned MWCNTs. Top (h) and middle (i) portions of ' $\mathrm{T}$ ' with aligned MWCNTs. All long black arrows indicate the alignment direction.

stainless-steel spatula. Additional manual marks made in a direction perpendicular to the marks scraped manually earlier and are indicated by long black and white arrows, respectively, shown in figure 5a. FE-SEM analysis on the manual marks and the marks perpendicular to the manual marks reveals that the MWCNTs align along the direction of the force applied (figure $5 \mathrm{~b}$ and $\mathrm{c}$ ) similar to the alignment obtained in the above methods. The interface between both manual marks, where the alignment of MWCNTs is also perpendicular to each other and is clearly visible and is indicated by the long arrow marks as shown in figure $5 \mathrm{~d}$. It is therefore demonstrated in this work that, MWCNTs can be aligned and easily manipulated to any direction by applying physical forces.

MWCNTs were densely packed in the aligned region and it is found to be $\sim 28$ MWCNTs $\mu \mathrm{m}^{-1}$. Along with MWCNTs there are few budding MWCNTs which are terminated before their complete growth, polyhedral nanoparticles and nickel particles are also present. Nickel is used as a catalyst to favour the formation of MWCNTs during the arc discharge process and the formation of MWCNTs has been described elsewhere. ${ }^{19,20}$

\subsection{Purification of the raw soot}

The nickel particles present in the soot were removed by immersing the soot in $6 \mathrm{M} \mathrm{HCl}$ solution at room temperature for $10 \mathrm{~h}$ and neutralized with distilled water to remove the acid content. SEM micrograph of the manually aligned region in the letter 'I' (figure 2e) in SE mode before purification shows nickel particles represented by a circle, which is absent after purification in the same region shown in figure $6 a$ and $b$, respectively. It is seen that the aligned regions are not disturbed after purification. BSE mode with EDS analysis further confirms the presence and removal of nickel before and after purification as shown in figure $6 \mathrm{~d}$ and e, respectively. The alignment of the MWCNTs is undisturbed even after the removal of nickel in the manually aligned and in-situ aligned regions of the soot as confirmed by FE-SEM analysis as shown in figure $6 \mathrm{c}$ and $\mathrm{f}$, 
(a)

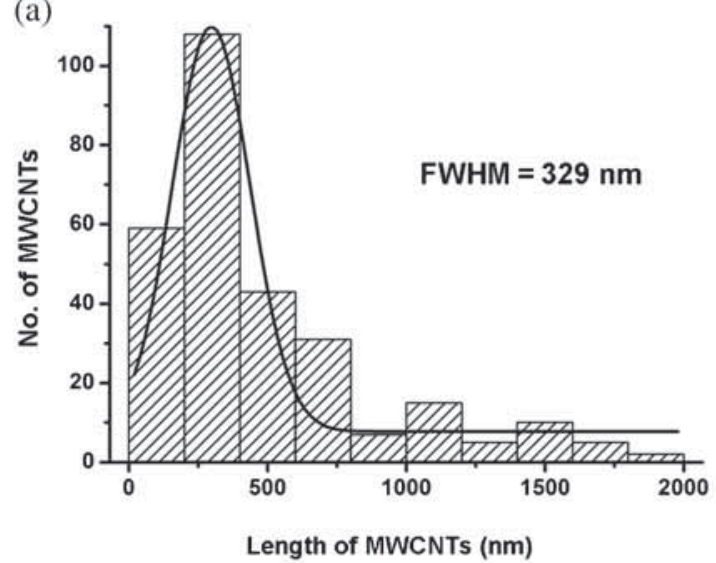

(c)

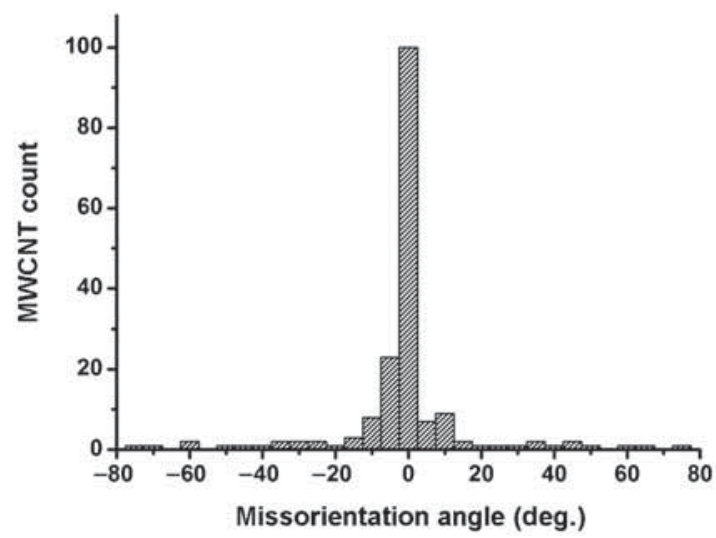

(b)

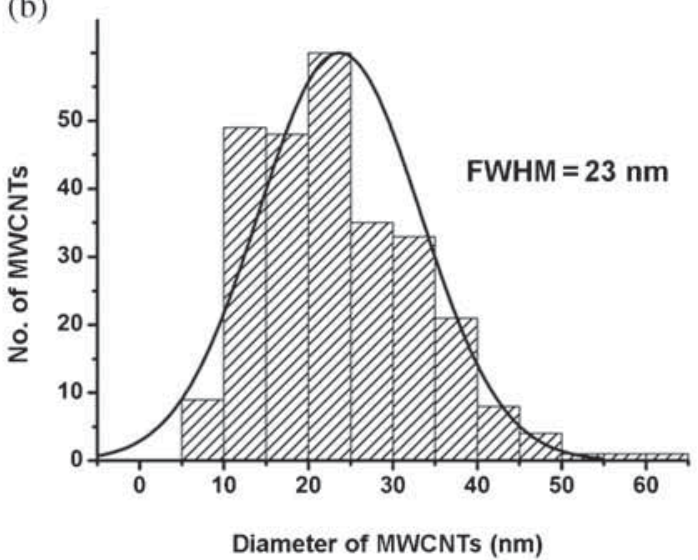

(d)

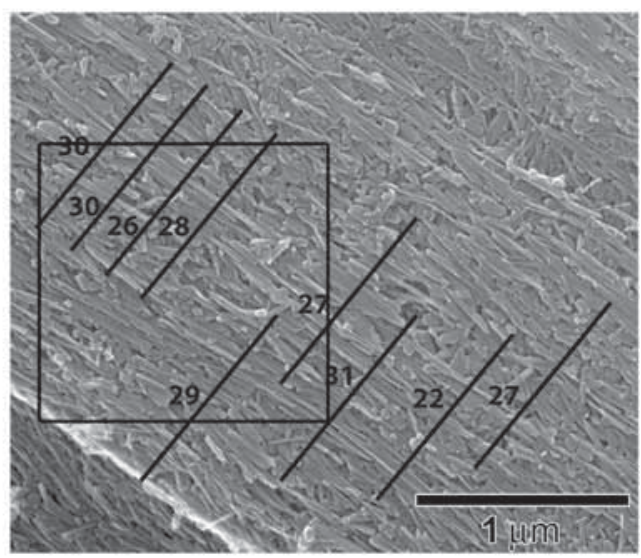

Figure 3. Distribution of length of MWCNTs, FWHM is $329 \mathrm{~nm}$ from Gaussian fit (a), diameter of MWCNT, FWHM is $23 \mathrm{~nm}$ from Gaussian fit (b), misorientaion of MWCNTs in the aligned region (c) (shown in d). Number of MWCNTs packed per $\mu \mathrm{m}$ is $\sim 28(\mathbf{d})$, indicated by the black lines used for measurements in the aligned region of the middle portion of the letter 'I' as shown in $\mathbf{c}$.

respectively. This opens the possibility that the process demonstrated in this work can generate aligned CNTs without nickel, even though the synthesis involves the use of nickel.

\subsection{Raman spectroscopy analysis of CNTs}

Raman spectroscopic analysis was performed on the aligned and unaligned regions of the unpurified and purified surfaces of the soot, where the SEM analysis were carried out, as shown in figure 7. A band at $\sim 1335 \mathrm{~cm}^{-1}$ arises due to the breathing of hexagons known as defect band D..$^{21}$ A sharp and narrow high intensity Raman frequency band arises at $\sim 1570 \mathrm{~cm}^{-1}$, also known as graphitic band (G), due to the $\mathrm{E}_{2 \mathrm{~g}}$ stretching mode vibration of neighbouring carbon atoms moving in opposite directions in a graphene sheet. The intensity of the D band is the signature of the defects present in the sample, which can be quantified by the ratio of intensity of the $\mathrm{D}$ band to the intensity of the $\mathrm{G}$ band $\left(I_{\mathrm{D}} / I_{\mathrm{G}}\right)$. The defect densities of the purified and unpurified samples are relatively lesser (10.7-33.6\%), compared to the MWCNTs grown by other methods, which implies the presence of the nanotubes with higher quality. The quality of the nanotubes is further confirmed by TEM analysis. There is a significant decrease in the intensity of the defect band (D) in the unaligned region compared with the aligned region in both unpurified (9.3\%) (figure $7 \mathrm{a}$ and $\mathrm{b}$ ) and purified (16.7\%) (figure 7c and d) samples. The intensity of the $\mathrm{D}$ band in the unaligned and aligned regions of the unpurified samples is lower (10.7 and 20\%) than the purified samples (16.9 and 33.6\%). The reduction in peak intensity occurs due to the presence of nickel particles that covered the defective regions (figure $7 \mathrm{a}$ and d) such as the tips of the nanotubes and the other nanoparticles, which relatively reduces the intensity of the $\mathrm{D}$ band in the unpurified samples compared to the purified samples. The increase in the D band intensity in the purified samples is an additional confirmation of the purification of the soot. Since TEM examination reveals no serious damage in the nanotubes, the increase in the D band intensity in the aligned region may not be possibly due to the generation of defects during the application of forces for the nanotubes alignment. It could be due to the exposure of the defects such as pentagon and heptagon present in the tips of the nanotubes oriented along the aligning direction. The visibility of the nanotube tips is 


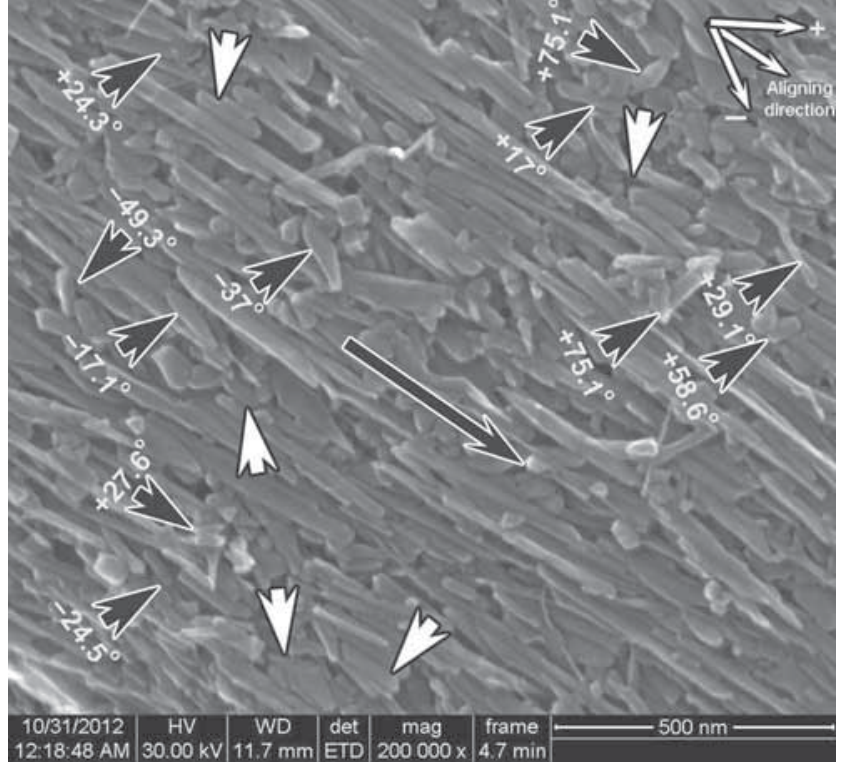

Figure 4. FE-SEM micrograph of the aligned MWCNTs portion shown in figure $3 \mathrm{~d}$ at higher magnification shows, the deviation of the shorter MWCNTs and the angle of deviation (17-75.1 ${ }^{\circ}$ ) from the principal aligning direction are indicated by black arrows and the shorter nanotubes aligned due to the forces exerted by the neighbouring MWCNTs are indicated by white arrows. more in the aligned region than the unaligned region due to the dispersion, which causes increase in the $\mathrm{D}$ band intensity in both cases of purified and unpurified samples. The difference in the defect ratios between the unaligned and aligned surfaces is extensively dependent on the aspect ratios of the nanotubes and number of the nanoparticles present. The difference will be lesser in the case of the increase in number of nanoparticles, which are already exposing the defects on the surface to the laser radiation and will be having negligible effects on the intensity of the defect peak after alignment. A second-order band at $\sim 2665 \mathrm{~cm}^{-1}$ is the overtone of D mode named as $\mathrm{G}^{\prime}$. The other weak second-order bands appear at 2425, 2905 and $3200 \mathrm{~cm}^{-1}$ are due to the phonon combination of $\mathrm{G}$ and $860 \mathrm{~cm}^{-1}$, overtone of $1450 \mathrm{~cm}^{-1}$ and overtone of $\mathrm{G}$ modes, respectively. $^{22}$

\subsection{XRD analysis on the CNTs soot surface}

The XRD pattern of the aligned regions of the soot is similar to graphite as shown in figure 8 . The presence of (002) peak corresponds to the graphitic carbon and the asymmetry in the $(h k 0)$ peaks is due to the curvature of the nanotubes, ${ }^{23}$ indicative of the presence of the MWCNTs in the sample. The XRD pattern of the soot shows similar characteristics to (a)
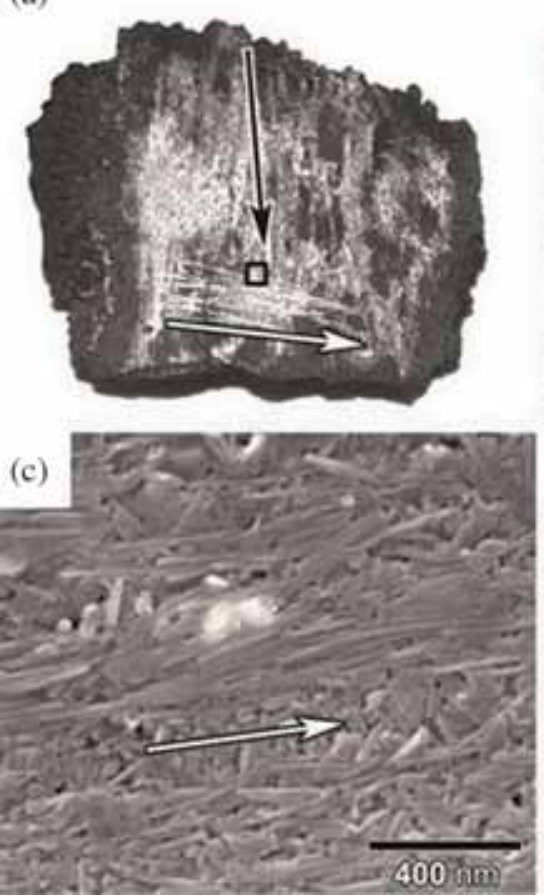

(b)
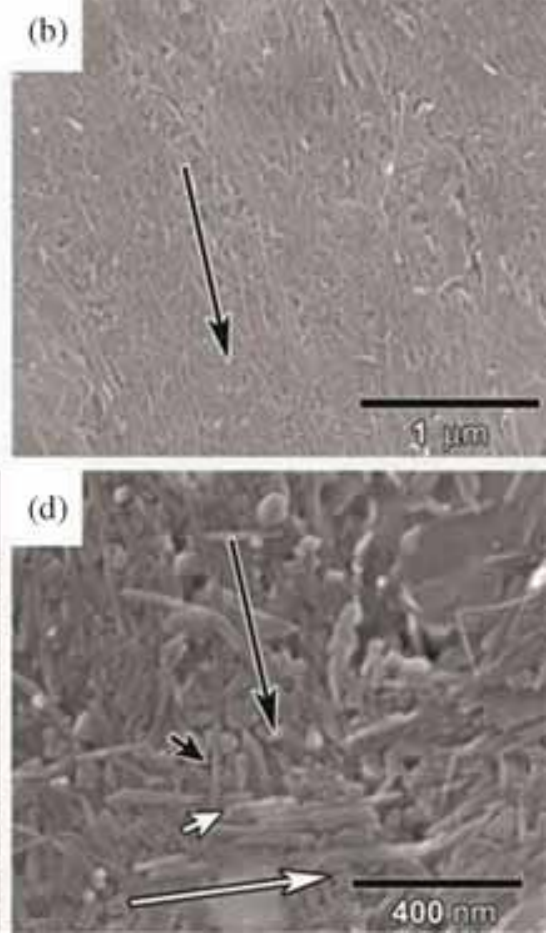

Figure 5. FE-SEM micrographs of as-synthesized soot with manual markings perpendicular to each other indicated by black and white arrows, respectively (a). Alignment of MWCNTs in the directions indicated by the black (b) and white (c) arrows in a. Interface between orthogonal scrape marks indicated by a square (d) in a. All long and short arrows indicate the alignment direction and MWCNTs, respectively. 

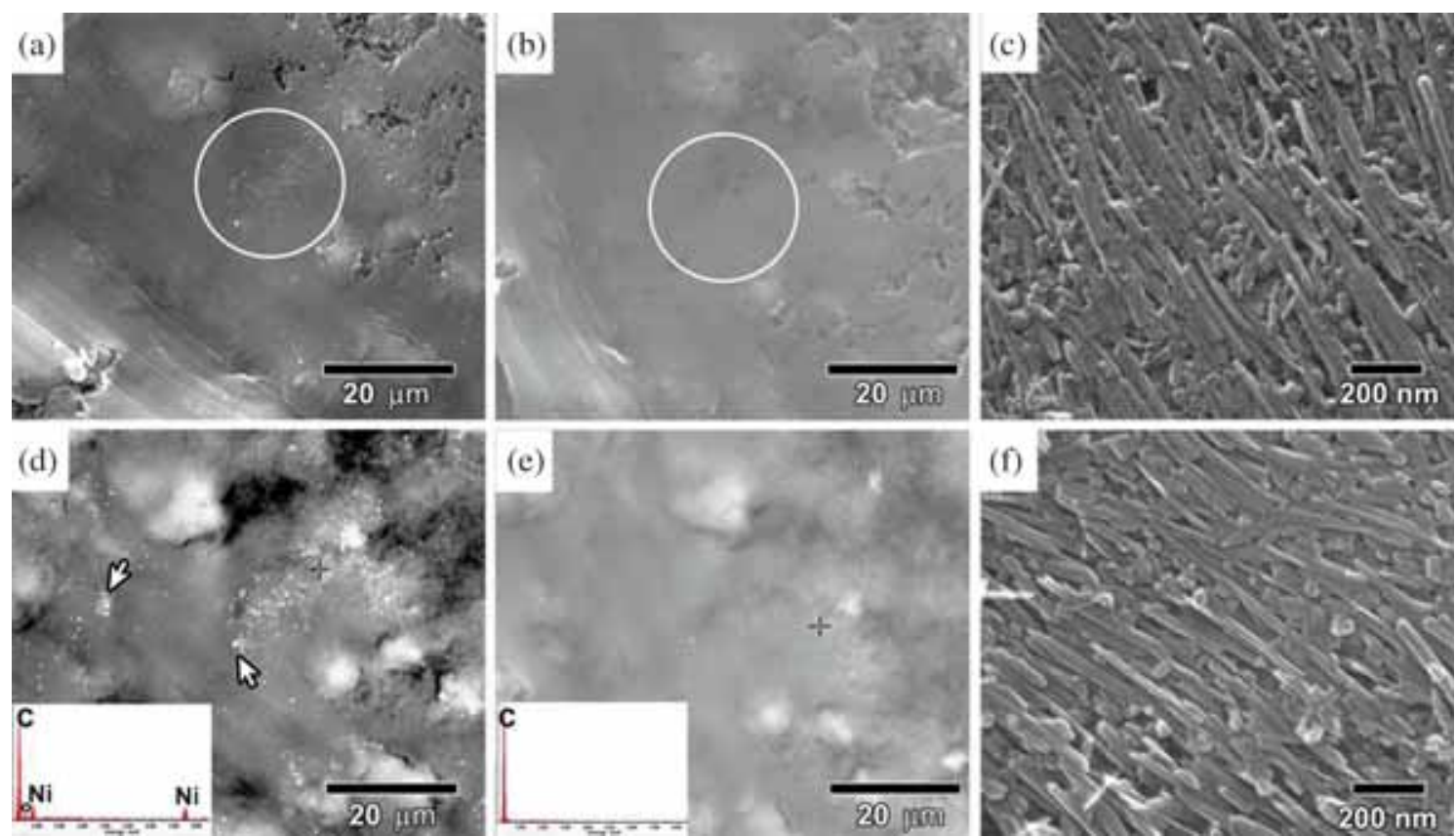

Figure 6. FE-SEM micrographs indicate the presence of nickel (encircled) in the region of middle portion of the inscription 'I' (figure 3e), before purification in SE mode (a); removal of nickel after purification in SE mode (b), micrograph of the encircled region in $\mathbf{b}$ shows that the alignment is retained (c). Presence and removal of nickel before and after purification ( $\mathbf{d}$ and $\mathbf{e}$ ), respectively, in BSE mode. Corresponding EDS analysis shows the presence of nickel in the inset. FE-SEM micrograph of an in-situ aligned region after purification (f).

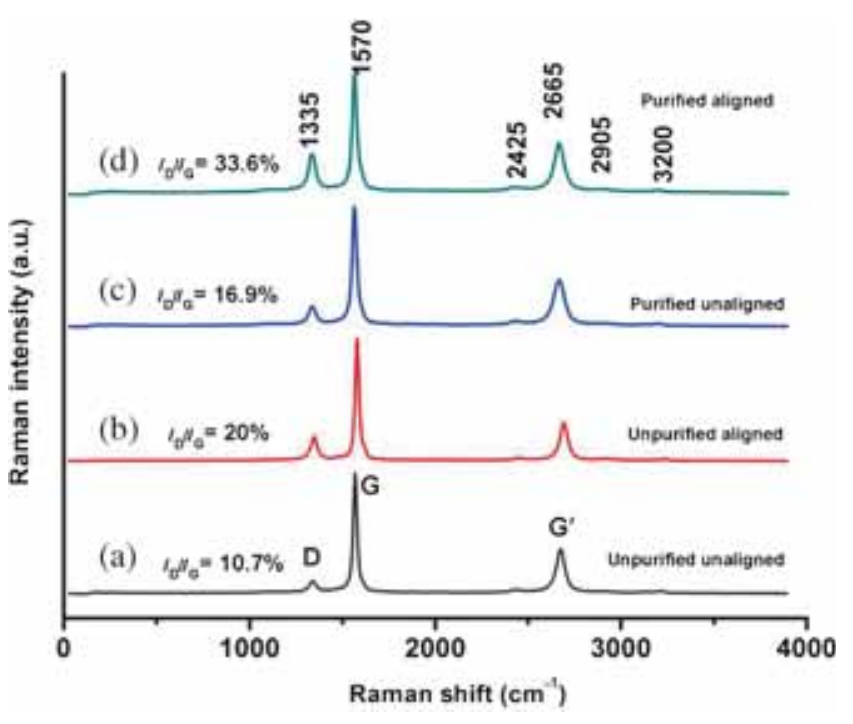

Figure 7. Raman spectroscopy analyses on the regions, middle of the 'I' (arrow b-aligned) and near the 'I' (label a-unaligned) shown in figure 3e: unpurified and unaligned (a), unpurified and aligned (b), purified and unaligned (c) and purified and aligned (d).

those of the soots prepared under a nitrogen atmosphere. ${ }^{17}$ The calalytic nickel present in the soot is removed after purification which is indicated by * (figure 8a) and is confirmed with a diminishing nickel peak in the purified sample (figure 8b).

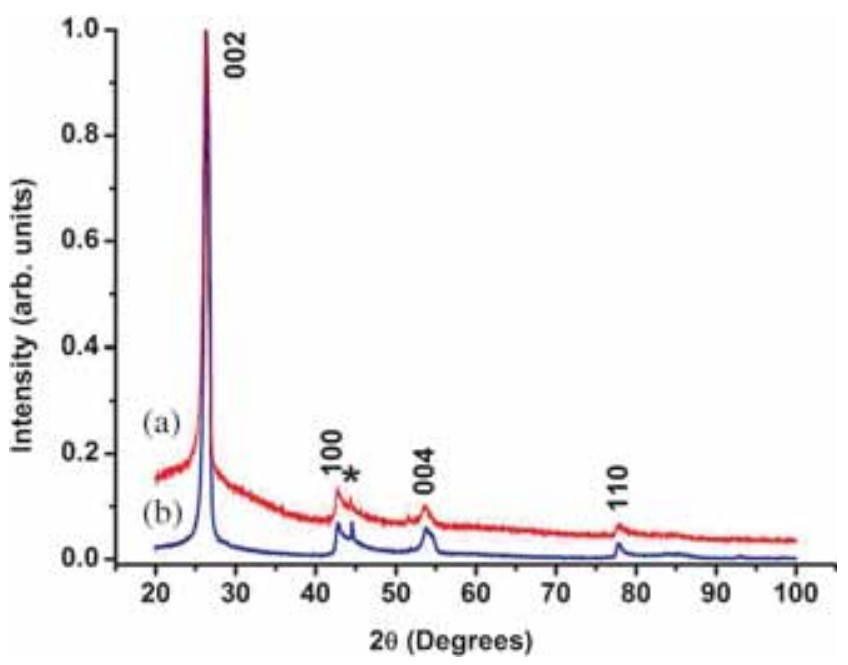

Figure 8. XRD pattern of the aligned soot surface before purification (a) and after purification (b). Diffraction peaks corresponding to nickel is indicated by $*$.

\subsection{TEM analysis of the MWCNTs in soot}

TEM analysis of the MWCNTs present in the as-synthesized soot surface shows straight tubular structures with few defects indicated by a white arrow shown in figure 9a. There are no additional defects created on the MWCNTs and these are not damaged after the application of the forces during 

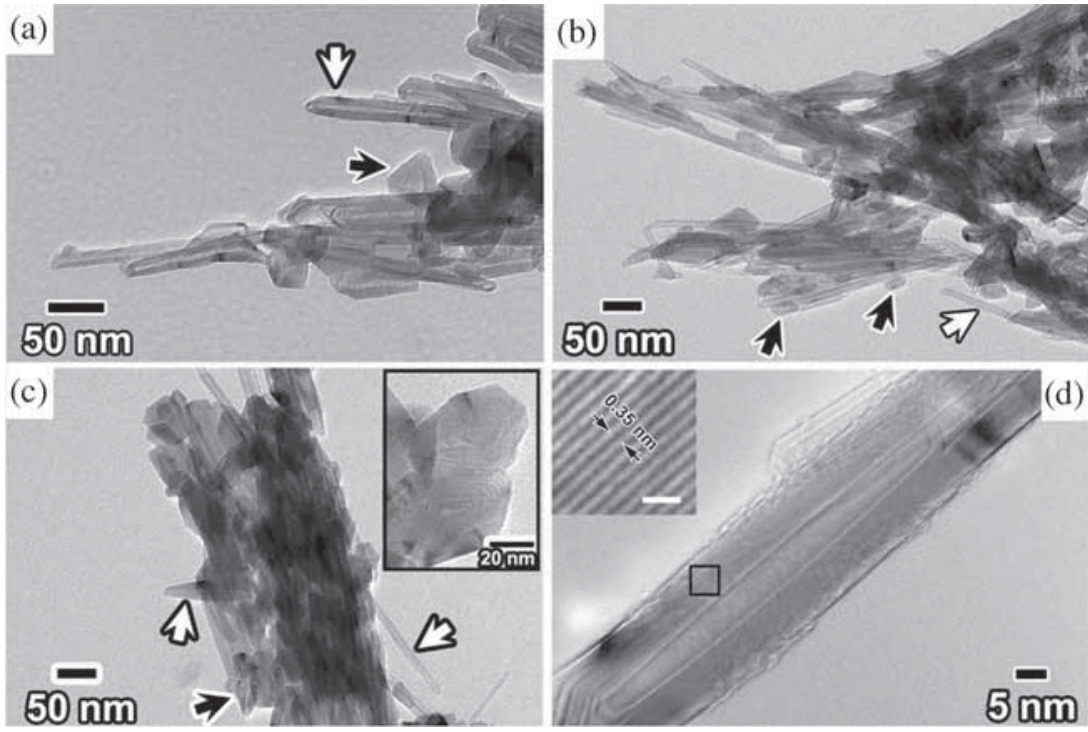

Figure 9. TEM micrographs of as-synthesized MWCNTs in the unaligned region (a), MWCNTs in the aligned region before purification (b), MWCNTs in the aligned region after purification (c). Inset shows the budding nanotubes. High-resolution micrograph showing lattice fringes (inset) and inter-tube distances in a MWCNT taken from the purified and aligned region in the soot $(\mathbf{d})$. The scale bar in the inset corresponds to $1 \mathrm{~nm}$. All white and black arrows indicate MWCNTs and budding nanotubes, respectively.

the in-situ and ex-situ aligning, which is confirmed by several TEM micrographs of the samples collected from the scratched surface as shown in figure $9 \mathrm{~b}$. No morphological changes occur on the nanotubes after the purification procedures carried out as shown in figure 9c. High-resolution TEM micrograph of a MWCNT (figure 9d) shows lattice fringes, which indicate that the nanotubes are highly graphitized. The inter-tube distance is calculated as $0.35 \mathrm{~nm}$, which is slightly higher than the interplanar spacing of the graphite due to the curvature of the nanotubes, ${ }^{24}$ as shown in the inset of figure 9d. The average length and diameter of MWCNTs calculated from TEM micrographs are in agreement with the FE-SEM results. Along with longer MWCNTs there are a few buds of MWCNTs which were terminated before their complete growth, indicated by black arrows in figure $9 \mathrm{a}-\mathrm{c}$, are present in the soot.

\subsection{Estimation of minimum force required to manipulate a bunch of MWCNT by nanoscratch testing}

The minimum force required to align the MWCNTs was estimated using nanoscratch test by manipulating the aligned MWCNTs on the surface of the soot. Scratch marks were made on the surface of the soot by applying 1000, $750 \mu \mathrm{N}$ (figure $10 \mathrm{a}-\mathrm{c}$ ) and $500 \mu \mathrm{N}$ (figure $10 \mathrm{~d}-\mathrm{f}$ ) forces up to a length of $20 \mu \mathrm{m}$. The MWCNTs were manipulated to the aligning direction by applying forces 1000 and $750 \mu \mathrm{N}$, and the alignment of the MWCNTs was confirmed using FE-SEM analysis. The direction of alignment of MWCNTs before and after manipulation is indicated by white and black arrows, respectively. A large portion of the MWCNTs is not manipulated when the force is reduced to $500 \mu \mathrm{N}$ (figure $10 \mathrm{e})$. When the load is applied, it will indent the soft aligned soot surface and the tip will be dragged up to a distance of $20 \mu \mathrm{m}$ which manipulates the nanotubes along the direction of the force applied. During this process, some MWCNTs are stuck on the sides of the tip and will be left over the manipulated regions in the scratch marks. It is also possible that the tip can drag and manipulate the loosely held MWCNTs in the aligned region along the direction of the force applied. Hence, the minimum force required to manipulate the MWCNTs is $\sim 750 \mu \mathrm{N}$ or should be $>500 \mu \mathrm{N}$, which is confirmed from the FE-SEM micrographs shown in figure 10.

\subsection{Calculations on energy and force required to manipulate a CNT}

The energy required to manipulate a gram of MWCNTs and the force required to manipulate a single MWCNT can be calculated based on the minimum force of $750 \mu \mathrm{N}$ used during the nanoscratch experiment. The experiments conducted did not resolve the value further between the 500 and $750 \mu \mathrm{N}$, and hence $750 \mu \mathrm{N}$ was identified as the minimum force required for aligning a collection of nanotubes within the framework of the nanoscratch technique.

In order to calculate the energy required to manipulate $1 \mathrm{~g}$ of MWCNTs $\left(E_{\mathrm{g}}\right)$ and the force required to manipulate a single MWCNT $\left(f_{\mathrm{M}}\right)$, it is necessary to calculate the mass of a MWCNT $\left(M_{\mathrm{M}}\right)$ and the number of MWCNTs aligned by a scratch $\left(N_{\mathrm{A}}\right)$. To calculate $M_{\mathrm{M}}$, it is necessary to first calculate the number of concentric tubes $n$, present in the MWCNT and the number of carbon atoms in the MWCNT 

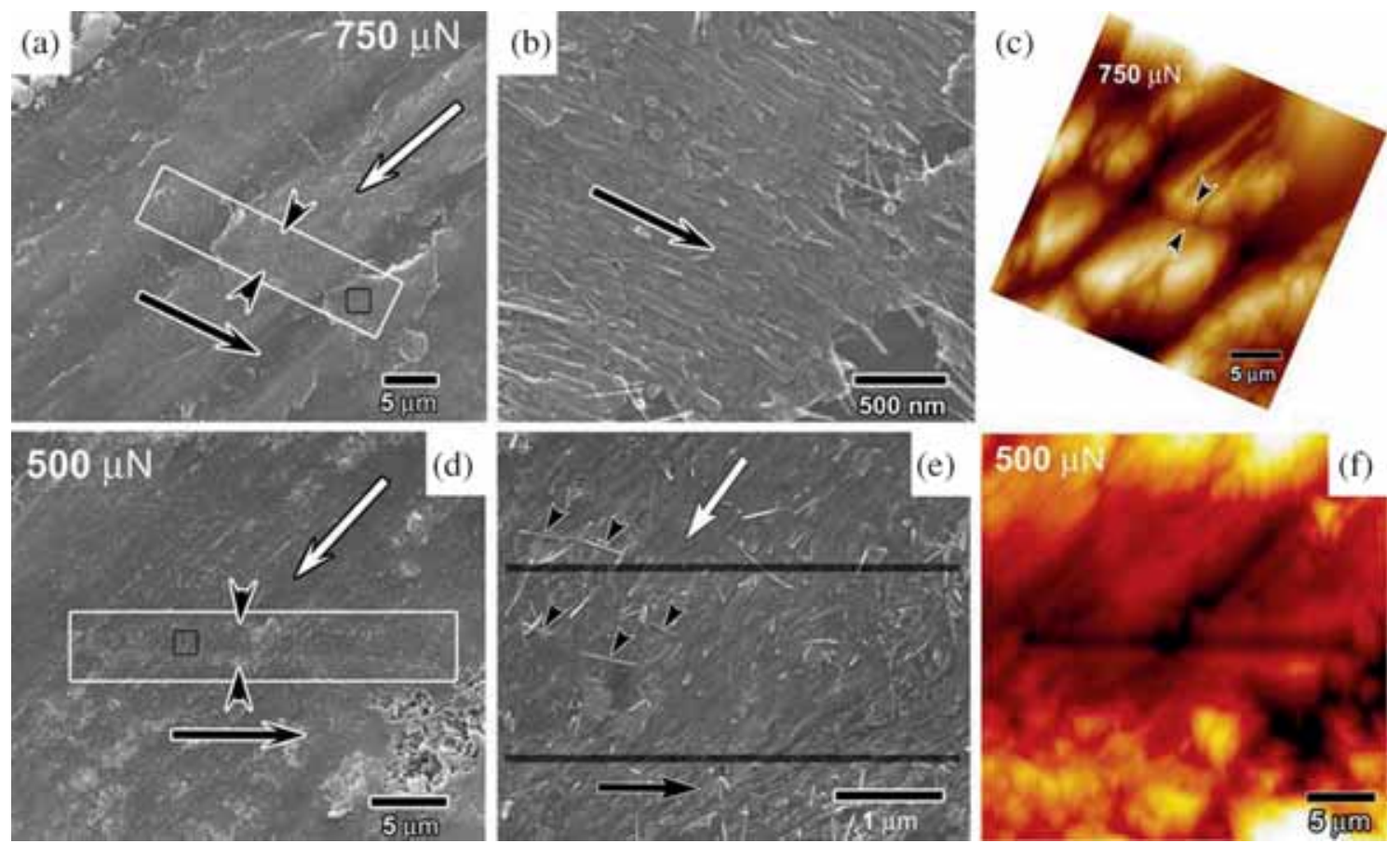

Figure 10. Scraped and manipulated directions are indicated by white and black arrows, respectively. FE-SEM micrograph of the scratch mark made at $750 \mu \mathrm{N}$ force across the aligned region at lower magnification (a), magnified portion of the scratch mark indicated by a black box shows the alignment of nanotubes along the scratching direction in a large white box portion (b) corresponding SPM micrograph of the scratch mark made at $750 \mu \mathrm{N}$ force (c). Lower and higher magnification FE-SEM micrographs of the manipulated surface with $500 \mu \mathrm{N}$ force, respectively (d and e). Corresponding SPM micrograph of the scratch mark made at $500 \mu \mathrm{N}$ force (f). Tailless black arrows in e indicate a few aligned nanotubes along the direction of the scratch.

$\left(N_{\mathrm{M}}\right) . N_{\mathrm{M}}$ will further depend on the number of carbon atoms in a single concentric tube of the MWCNT $\left(N_{\mathrm{S}}\right)$. These parameters are calculated below, leading up to calculation of the energy and force required to manipulate a MWCNT.

3.8a The number of concentric tubes $n$, present in the MWCNT: A MWCNT with inner tube diameter $\left(d_{\text {int }}\right)$ of $37 \AA$ $(3.7 \mathrm{~nm})$, inter-tube distance $\left(d_{\mathrm{itd}}\right)$ of $3.5 \AA(0.35 \mathrm{~nm})$, length $(L)$ of $4810 \AA(481 \mathrm{~nm})$, average diameter of a MWCNT $\left(d^{\prime}\right) 240 \AA$ ( $24 \mathrm{~nm}$ ), and total number of individual tubes $(n)$ is illustrated in figure 11a. The values of $d_{\mathrm{int}}, d_{\mathrm{itd}}, d^{\prime}$ and $L$ are assigned to the calculations based on the FE-SEM and TEM results.

The inner tube diameter of MWCNTs ranges from 0.4 to $7 \mathrm{~nm}$. Hence, the average inner tube diameter is

$$
d_{\text {int }}=\frac{0.4+7}{2} \mathrm{~nm}=3.7 \mathrm{~nm}=37 \AA .
$$

Number of individual nanotubes $(n)$ present in a MWCNT with outer tube diameter $d^{\prime}$, inner tube diameter $d_{\text {int }}$, and inter-tube distance $d_{\text {itd }}$ is calculated from the formula

$$
n=\frac{d^{\prime}-d_{\text {int }}}{2 \times d_{\text {itd }}}=\frac{240-37}{2 \times 3.5}=29+1 \quad \text { (inner tube) }=30 .
$$

3.8b Number of carbon atoms in a single concentric tube of the MWCNT $\left(N_{S}\right)$ :

Surface area of a nanotube $=\pi d_{\mathrm{i}} L$,

where

$d_{\mathrm{i}}$ is the diameter of an individual nanotube in a MWCNT and $L$ the length of the nanotube.

Area per hexagon of side ' $\mathrm{a}$ ' $(1.42 \AA)=\frac{3 \sqrt{3} \times a^{2}}{2}$.

Since, the effective number of carbon atoms per hexagon in a single nanotube is 2 , the total number of carbon atoms $\left(N_{\mathrm{s}}\right)$ in a single tube of diameter $d_{\mathrm{i}}$ and length $L$ is calculated as

$$
\begin{gathered}
N_{\mathrm{s}}=\frac{\pi d_{\mathrm{i}} L}{\left(3 \sqrt{3} a^{2} / 2\right)} \times 2=\left(\frac{4 \pi L}{3 \sqrt{3} a^{2}}\right) \\
\times d_{\mathrm{i}}=5768.9 \times 10^{10} \times d_{\mathrm{i}} .
\end{gathered}
$$$$
N_{\mathrm{s}}=\text { (Area of the nanotube/area of the hexagon) }
$$$$
\times \text { number of carbon atoms per hexagon, }
$$ 


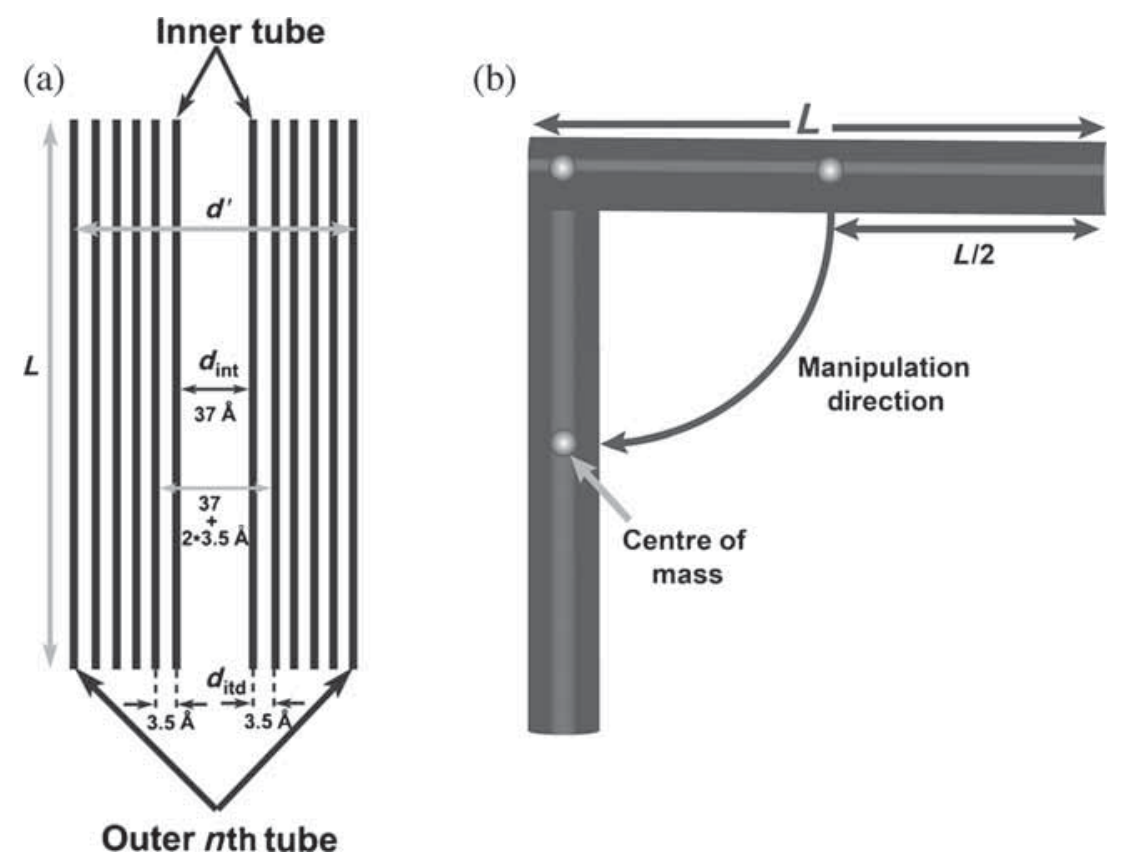

Figure 11. Illustration of a MWCNT with an average inner tube diameter $37 \AA$, intertube distance $3.5 \AA$ with $n$ number of outer tubes (a) and manipulation and centre of mass of the nanotube $(\mathbf{b})$.

$3.8 \mathrm{c}$ Number of carbon atoms in the MWCNT $\left(N_{M}\right)$ : Equation (2) is valid for a single nanotube with diameter $d_{\mathrm{i}}$. However, a MWCNT is composed of several individual concentric nanotubes with varying diameters from the inner most nanotube to the outer most nanotube. Since the inter-tube distance is calculated as $\sim 3.5 \AA$, the immediate nanotube next to the inner most nanotube will be having a diameter of the sum of the diameter of the inner most nanotube $(37 \AA)$ and twice the product of the inter-tube distance (3.5 $\AA$ ). Similarly, if a MWCNT contains ' $n$ ' nanotubes with a varying diameter $d_{\mathrm{i} 1}, d_{\mathrm{i} 2}, d_{\mathrm{i} 3}, \ldots d_{\mathrm{in}}$, then, sum of the diameters of all the individual nanotubes present in a MWCNT $\left(D_{\mathrm{n}}\right)$ can be calculated as shown in

$D_{\mathrm{n}}=\left[d_{\mathrm{int}}+d_{\mathrm{i} 1}+d_{\mathrm{i} 2}+\cdots+d_{\mathrm{in}}\right]$,

$D_{\mathrm{n}}=\left(\begin{array}{l}d_{\text {int }}+\left(d_{\text {int }}+2 \times d_{\text {itd }}\right)+\left(d_{\text {int }}+4 \times d_{\text {itd }}\right)+\cdots+ \\ \left(d_{\text {int }}+\left(2 \times d_{\text {in }}\right) \times d_{\text {itd }}\right) .\end{array}\right)$

Hence, $d_{\mathrm{i}}$ for a nanotube with single wall becomes $D_{\mathrm{n}}$ for a MWCNT and the total number of carbon atoms present in the MWCNT can be calculated as shown. The total number of carbon atoms in a MWCNT $\left(N_{\mathrm{M}}\right)$, can be calculated as, as a product of (2) and (3)

$$
N_{\mathrm{M}}=N_{\mathrm{s}} \times D_{\mathrm{n}} .
$$

Substituting the values of $d_{\text {int }}$ and $d_{\text {itd }}$, then

$$
N_{\mathrm{M}}=5768.9 \times 10^{10} \times\left(\begin{array}{l}
37+(37+2 \times 3.5)+(37+ \\
4 \times 3.5)+\cdots+(37+58 \times 3.5)
\end{array}\right),
$$

$$
N_{\mathrm{M}}=5768.9 \times 10^{10} \times\left(\begin{array}{c}
(37 \times 30)+2 \times 3.5(1+ \\
2+3+4+\cdots+29)
\end{array}\right) .
$$

Since

$$
(1+2+3+4+\cdots+n)=\sum_{i=1}^{n} \frac{n(n+1)}{2} .
$$

Since, $n=29$ without the inner nanotube, then it becomes

$$
\begin{aligned}
N_{\mathrm{M}}= & 5768.9 \times 10^{10} \times\left((37 \times 30)+2 \times 3.5\left(\frac{29 \times 30}{2}\right)\right) \\
& \times 10^{-10} .
\end{aligned}
$$

The total number of carbon atoms in a MWCNT is calculated as shown as

$$
N_{\mathrm{M}}=2.4 \times 10^{7} \text { carbon atoms. }
$$

3.8d Mass of a MWCNT $\left(M_{M}\right)$ : Since $12 \mathrm{~g}$ of carbon contains Avogadro number of carbon atoms, mass of the MWCNT can be calculated as

$$
M_{\mathrm{M}}=N_{\mathrm{M}} \times \frac{12}{6.023 \times 10^{23}}=4.78 \times 10^{-16} \mathrm{~g} .
$$

3.8e Number of MWCNTs aligned by a scratch $\left(N_{A}\right)$ : From the FE-SEM analysis, width of the scratch $=4 \mu \mathrm{m}$, length of the scratch $=20 \mu \mathrm{m}$; therefore area of the scratch $=80 \mu \mathrm{m}^{2}$. Number of MWCNTs packed per micrometre of width $=28$, i.e., in $1 \mu \mathrm{m}$ width there are $\sim 28$ nanotubes present. Average length of the nanotube is $481 \mathrm{~nm}$ or 
$0.481 \mu \mathrm{m}$. Therefore, total number of aligned MWCNTs in the scratch area $\left(N_{\mathrm{A}}\right)$ can be calculated from the FE-SEM micrographs as

$$
N_{\mathrm{A}}=\frac{80 \mu \mathrm{m}^{2}}{0.481 \mu \mathrm{m} \times 1 \mu \mathrm{m}} \times 28=4656 \text { MWCNTs. }
$$

3.8f Energy required to align a gram of nanotubes: Work done to manipulate the MWCNT by scratching the surface can be termed as a product of the force $(F)$ applied to scratch the surface to the distance moved $(d)$ by the centre of mass (figure 11b) of the MWCNT along the direction of the force applied.

$$
\text { Work done }=\text { Force } \times \text { Distance }=F \times d \text {. }
$$

$F=750 \times 10^{-6} \mathrm{~N}$, which is the force applied by the tip to align the MWCNTs,

$d=$ distance moved by the centre of mass of the MWCNT along the direction of the force applied is $L / 2$. Substituting the values of $F$ and $d$ then

$$
W=750 \times 10^{-6} \times\left(\frac{L}{2}\right)=1.8 \times 10^{-10} \mathrm{~J} .
$$

Work done or energy required per MWCNT to manipulate ( $W_{\mathrm{M}}$ ) can be calculated by dividing (5) by $N_{\mathrm{A}}$ is as shown below

$$
W_{\mathrm{M}}=\frac{W}{N_{\mathrm{A}}}=\frac{1.8 \times 10^{-10}}{4656}=38.65 \times 10^{-15} \mathrm{~J} / \mathrm{MWCNT} .
$$

Energy required to manipulate MWCNTs per gram: The number of aligned MWCNTs present on the scratched surface is 4656 and which cover an area of $80 \mu \mathrm{m}^{2}$. The number of MWCNTs $\left(N_{\mathrm{MW}}\right)$ present in $1 \mathrm{~g}$ of MWCNT is calculated as

$$
N_{\mathrm{MW}}=\frac{1}{M_{\mathrm{M}}}=\frac{1}{4.78 \times 10^{-16}}=2.09 \times 10^{15} \text { nanotubes. }
$$

The area covered $\left(A_{\mathrm{M}}\right)$ by $1 \mathrm{~g}$ of nanotubes $\left(N_{\mathrm{MW}}\right)$ can be calculated as

$$
\begin{aligned}
A_{\mathrm{M}} & =\frac{\text { Area of the scratch }}{N_{\mathrm{A}}} \times N_{\mathrm{MW}}=\left(\frac{80}{4656}\right) \times 2.09 \times 10^{15} \\
& =3.6 \times 10^{13} \mu \mathrm{m}^{2}=36 \mathrm{~m}^{2} .
\end{aligned}
$$

Energy required to manipulate a gram of nanotubes $\left(E_{\mathrm{g}}\right)$ can be calculated from the product of the energy to manipulate a single nanotube and the number of nanotubes in a gram, as shown below

$$
E_{\mathrm{g}}=W_{\mathrm{M}} \times N_{\mathrm{MW}}=38.65 \times 10^{-15} \times 2.09 \times 10^{15}=80.78 \mathrm{~J} .
$$

$3.8 \mathrm{~g}$ Force required to manipulate a single MWCNT: Force required to manipulate a single MWCNT $\left(f_{\mathrm{M}}\right)$ calculated as shown in equation (8), by equating the total work done during the scratch to the work on a single nanotube multiplied with the total number of nanotubes that were manipulated during the scratching.

$$
\begin{aligned}
f_{\mathrm{M}} & =\frac{F_{\mathrm{t}} \times L_{\mathrm{s}}}{(L / 2) \times N_{\mathrm{A}}}, \\
f_{\mathrm{M}} & =\frac{750 \times 10^{-6} \times 20 \times 10^{-6}}{(481 / 2) \times 10^{-9} \times 4656} \\
& =13.3 \times 10^{-6} \mathrm{~N}=13.3 \mu \mathrm{N},
\end{aligned}
$$

where $f_{\mathrm{M}}$ is the force required to align a single MWCNT, $L / 2$ the distance moved due to the force applied on a MWCNT, $N_{\mathrm{A}}$ the total number of aligned MWCNTs, $F_{\mathrm{t}}$ the force applied by the tip on the surface of the soot, $L_{\mathrm{S}}$ the total length of the scratch mark.

By this calculation, from the observations and measurements the minimum force required to align a MWCNT using the nanoscratch technique is $\sim 13 \mu \mathrm{N}$ and the energy required to align a gram of nanotubes is $\sim 80.78 \mathrm{~J}$.

\section{Conclusions}

Densely packed and horizontally aligned MWCNTs, are obtained using a rotating cathode arc discharge method in the open air by the application of simple physical forces. The alignment can also be controlled and manipulated to directions of interest by applying physical forces both during as well as after synthesis. The presence of nickel is identified in the soot and removed without disturbing the alignment of MWCNTs. It is a simple and low cost method of producing well aligned and densely packed MWCNTs in a single step with a relatively higher production rate compared with other techniques. Analysis of the alignment process has been carried out using the nanoscratch technique. The minimum force required to align the nanotubes on the surface of the soot is seen to be greater than $500 \mu \mathrm{N}$. Using a model to understand the alignment process, and based on the data obtained from the nanosratch technique, the minimum force required aligning a single MWCNT is determined to be $13.3 \mu \mathrm{N}$, and the energy required to align a gram of nanotubes is estimated to be $80.78 \mathrm{~J}$. The alignment of MWCNTs obtained in a single step during synthesis in open air, without the need for a controlled atmosphere, opens up the possibilities of production of aligned nanotubes in a larger scale for commercial applications.

\section{References}

1. Iijima S 1991 Nature 35456

2. Joshi R, Engstler J, Nair P K, Haridoss P and Schneider J J 2008 Diam. Relat. Mater. 17913

3. Cheng H M, Li F, Su G, Pan H Y, He L L, Sun X and Dresselhaus M S 1998 Appl. Phys. Lett. 723282 
4. Thess A, Lee R, Nikolaev P, Dai H, Petit P, Robert J, Xu C, Lee Y H, Kim S G, Rinzler A G, Colbert D T, Scuseria G E, Tománek D, Fischer J E and Smalley R E 1996 Science 273 483

5. Iijima $S$ and Ichihashi T 1993 Nature $\mathbf{3 6 3} 603$

6. Ando Y, Zhao X, Inoue S and Iijima S 2002 J. Cryst. Growth 237-239 1926

7. Hong S and Myung S 2007 Nature 2207

8. Choi W B, Bae E, Kang D, Chae S, Cheong B H, Ko J H, Lee E and Park W 2004 Nanotechnology 15 S512

9. Ajayan P M, Stephan O, Colliex C and Trauth D 1994 Science 2651212

10. Ajayan P M 1995 Adv. Mater. 7489

11. Jin L, Bower C and Zhou O 1998 Appl. Phys. Lett. 731197

12. Lee C J, Kim D W, Lee T J, Choi Y C, Park Y S, Lee Y H, Choi W B, Lee N S, Park G S and Kim J M 1999 Chem. Phys. Lett. 312461

13. Lu J, Miao J, Xu T, Yan B, Yu T and Shen Z 2011 Nanotechnology 22265614
14. Orofeo C M, Ago H, Ikuta T, Takahasi K and Tsuji M 2010 Nanoscale 21708

15. Hedberg J, Dong L and Jiao J 2005 Appl. Phys. Lett. 861

16. Correa-Duarte M A, Grzelczak M, Salgueiriño-Maceira V, Giersig M, Liz-Marzán L M, Farle M, Sierazdki K and Diaz R 2005 J. Phys. Chem. B 10919060

17. Joseph B A, Ramakrishnan S, Jain G and Haridoss P 2013 Carbon $\mathbf{5 5} 185$

18. Lu L and Chen W 2010 ACS Nano 41042

19. Harris P J F 2007 Carbon 45229

20. Gamaly E G and Ebbesen T W 1995 Phys. Rev. B 522083

21. Hiura H, Ebbesen T W, Tanigaki K and Takahashi H 1993 Chem. Phys. Lett. 202509

22. Dresselhaus M S, Dresselhaus G, Saito R and Jorio A 2005 Phys. Rep. 40947

23. Reznik D, Olk C H, Neumann D A and Copley J R D 1995 Phys. Rev. B $\mathbf{5 2} 116$

24. Kiang $\mathrm{C} \mathrm{H}$, Endo M, Ajayan P M, Dresselhaus G and Dresselhaus M S 1998 Phys. Rev. Lett. 811869 